\title{
Reorientation of Asymmetric Rigid Body Using Two Controls
}

\author{
Donghoon Kim, ${ }^{1}$ James D. Turner, ${ }^{1}$ and Henzeh Leeghim ${ }^{2}$ \\ ${ }^{1}$ Texas A\&M University, College Station, TX 77843-3141, USA \\ ${ }^{2}$ Chosun University, Gwangju 501-759, Republic of Korea \\ Correspondence should be addressed to Henzeh Leeghim; h.leeghim@gmail.com
}

Received 9 May 2013; Accepted 25 July 2013

Academic Editor: Mufid Abudiab

Copyright (C) 2013 Donghoon Kim et al. This is an open access article distributed under the Creative Commons Attribution License, which permits unrestricted use, distribution, and reproduction in any medium, provided the original work is properly cited.

\begin{abstract}
Most spacecrafts are designed to be maneuvered to achieve pointing goals. This is accomplished usually by designing a three-axis control system, which can achieve arbitrary maneuvers, where the goal is to repoint the spacecraft and match a desired angular velocity at the end of the maneuver. New control laws are required, however, if one of the three-axis control actuators fails. This paper explores suboptimal maneuver strategies when only two control torque inputs are available. To handle this underactuated system control problem, the three-axis maneuver strategy is transformed to two successive independent submaneuver strategies. The first maneuver is conducted on one of the available torque axes. Next, the second maneuver is conducted on the torque available plane using two available control torques. However, the resulting control law is more complicated than the general three-axis control law. This is because an optimal switch time needs to be found for determining the end time for the single-axis maneuver or the start time for the second maneuver. Numerical simulation results are presented that compare optimal maneuver strategies for both nominal and failed actuator cases.
\end{abstract}

\section{Introduction}

This work addresses the problem of finding suboptimal spacecraft maneuver control laws for handling underactuated system, with only two control torques available. Many researchers have considered controlling the attitude of rigid and flexible spacecrafts when all actuators are available. Many different control strategies have been introduced for handling the nominal three-axis control case [1-3]. For underactuated system control application, more limited literatures exist. For example, Tsiotras and Longuski [4] have considered the case designing control strategies for handling situations in which sensor and actuator failures limit the control options available for carrying out the original mission objectives. Keraï [5] has considered a more extreme case in which only a single control actuator is available but is shown to be uncontrollable, which is intuitively reasonable. Brockett [6] has shown that two controls can be made asymptotically stable about the origin. Tsiotras et al. [7-14] have further addressed the problem of stabilization of axis-symmetric spacecraft including tracking control laws. Others $[15,16]$ have presented approximate strategies that switch between two different control laws. Much of the later work has considered complex mathematical approaches for overcoming the underactuated spacecraft control problem. Recently, Kim and Turner [17] have suggested a simple way to handle the failure control problem by introducing a sequential maneuver approach that avoids exciting nonlinear coupling interaction effects in the equation of motion and attitude kinematics during suboptimal maneuvers. Analytically the problem leads to a high-dimensional optimization problem, where it is very important to specify accurate starting guesses [18]. The problem becomes computationally challenging, because of increased number of unknowns and constraints [19]. A major contribution to this work is the reduction of the number of unknowns and constraints through two successive maneuver strategies. Reducing three submaneuvers to two submaneuvers confirms that the suggested strategy requires less torque than the three-successive-maneuver strategy.

In this work, we address the problem of formulating and solving a rigorous nonlinear optimal control problem formulation for handling spacecraft maneuvers where actuator failures limit the number of control inputs to two axes. First, a single-axis maneuver is conducted to move the given attitude 
to a torque available plane. Then the general simultaneous maneuver is conducted for reorienting the spacecraft attitude. The necessary conditions are developed for two cases, such as control torque minimization and control torque-rate minimization. Unless the closed-loop control is considered, the second approach provides continuous control time histories to avoid an unexpected flexible body response $[20,21]$. The nonlinear necessary conditions are handled by introducing a multiple shooting method [22], which enforces both the terminal and interior boundary conditions that define the optimal solution.

An asymmetric rigid spacecraft math model with two control inputs is assumed. The control design objective is to avoid the axis where the actuator failure has occurred. With only two control inputs available, our strategy consists of carrying out two successive submaneuvers of unknown duration. For obtaining a suboptimal solution, an optimal switch time must be found. Provided numerical examples demonstrate that, if one fails to determine the optimal switch time, then the impact on the integrated value of the performance index is very significant. The full nonlinear set of necessary conditions is solved by introducing a multiple shooting method that is found to require $\sim 80$ iterations for convergence.

\section{Dynamics and Kinematics for a Rigid Body}

In general, the rotational dynamics equation of a rigid spacecraft is given by [1-3]

$$
\dot{\omega}=J^{-1}\left(-\left[\omega^{\times}\right] J \omega+\mathbf{U}\right)
$$

where $J \in \mathscr{R}^{3 \times 3}$ is the moment of inertia tensor for the spacecraft, which is assumed to be diagonal, $\boldsymbol{\omega} \in \mathscr{R}^{3}$ is the angular velocity vector of the spacecraft, and $\mathbf{U} \in \mathscr{R}^{3}$ is the control torque vector, and the cross-product matrix with a generic variable $\mathbf{k} \in \mathscr{R}^{3}$ is define as

$$
\left[\mathbf{k}^{\times}\right] \triangleq\left[\begin{array}{ccc}
0 & -k_{3} & k_{2} \\
k_{3} & 0 & -k_{1} \\
-k_{2} & k_{1} & 0
\end{array}\right]
$$

For describing an actuator failure condition, (1) is modified as [21]

$$
\dot{\boldsymbol{\omega}} \triangleq \mathbf{f}(\mathbf{u}, \boldsymbol{\omega})=J^{-1}\left(-\left[\boldsymbol{\omega}^{\times}\right] J \boldsymbol{\omega}+P \mathbf{u}\right),
$$

where $\mathbf{u} \in \mathscr{R}^{2}$ is the available control torque vector, and the control mapping matrix $P$ is defined as

$$
P \triangleq\left[\begin{array}{ll}
1 & 0 \\
0 & 1 \\
0 & 0
\end{array}\right]
$$

There are various parameters to describe attitude such as the quaternion, Euler angles, direction cosine matrix, modified Rodrigues parameters (MRPs), and so forth. Considering the number of parameters to describe the attitude and singularity issues, the MRPs are selected to define kinematic equations. The MRPs are defined in terms of the quaternion or the principal rotational elements as [23]

$$
\begin{gathered}
\boldsymbol{\sigma}=\frac{\boldsymbol{\rho}}{1+q_{4}} \\
\boldsymbol{\sigma}=\widehat{\mathbf{e}} \tan \frac{\Phi}{4}
\end{gathered}
$$

where the MRPs have a geometric singularity at $\Phi= \pm 2 \pi$ radians from $(5 b)$.

The kinematic differential equations for the MRPs can be expressed as

$$
\dot{\boldsymbol{\sigma}} \triangleq \mathbf{r}(\boldsymbol{\sigma}, \boldsymbol{\omega})=\frac{1}{4}[B(\boldsymbol{\sigma})] \boldsymbol{\omega},
$$

where

$$
[B(\boldsymbol{\sigma})] \triangleq\left(1-\boldsymbol{\sigma}^{T} \boldsymbol{\sigma}\right) I_{3 \times 3}+2\left[\boldsymbol{\sigma}^{\times}\right]+2 \boldsymbol{\sigma} \boldsymbol{\sigma}^{T}
$$

\section{Problem Formulation for Normal Control}

Two quadratic performance indices are introduced for defining the optimal control problem for the actuator mechanical failure maneuver case. Both the nominal and failed control actuator maneuvers are designed to achieve the threedimensional rigid body boundary conditions. Two related control formulations are presented: (i) quadratic penalties on control, leading to discontinuous control time histories, and (ii) quadratic penalties on control rate, leading to continuous control time histories. Both performance indices are defined as follows:

$$
\begin{aligned}
& \mathcal{J}^{\mathbf{u}} \triangleq \frac{1}{2} \int_{t_{0}}^{t_{f}} \mathbf{u}^{T} \mathbf{u} \mathrm{d} t \\
& \mathcal{J}^{\overline{\mathbf{u}}} \triangleq \frac{1}{2} \int_{t_{0}}^{t_{f}} \dot{\mathbf{u}}^{T} \dot{\mathbf{u}} \mathrm{d} t
\end{aligned}
$$

where the time derivative of control torque is defined as

$$
\dot{\mathbf{u}} \triangleq \mathbf{g}(\dot{\mathbf{u}}) \in \mathscr{R}^{2}
$$

As shown in the numerical results section, (8a) leads to a discontinuous control solution whereas (8b) leads to a continuous control solution. With only two control inputs available for the failed actuator case, one defines first singleaxis maneuver to move given attitude to controllable plane. By introducing two successive submaneuvers, one must define an unknown switch time between the first single-axis maneuver and the following maneuver.

3.1. Optimal Control Formulation. We seek a solution of (3) and (6) satisfying the prescribed terminal boundary conditions

$$
\begin{array}{cc}
\boldsymbol{\sigma}\left(t_{0}\right)=\boldsymbol{\sigma}_{0}, & \boldsymbol{\omega}\left(t_{0}\right)=\boldsymbol{\omega}_{0}, \\
\boldsymbol{\sigma}\left(t_{f}\right)=\boldsymbol{\sigma}_{f}, & \boldsymbol{\omega}\left(t_{f}\right)=\boldsymbol{\omega}_{f},
\end{array}
$$


where the 12 members of (10) are prescribed constants characterizing the attitude and angular velocity at the initial and final times. Defining the Hamiltonian for the system

$$
\mathscr{H}=\mathscr{L}+\xi^{T} \mathbf{r}+\boldsymbol{\mu}^{T} \mathbf{f},
$$

where the loss function $\mathscr{L}=\mathbf{u}^{T} \mathbf{u} / 2$ and the Lagrange multipliers are $\xi \in \mathscr{R}^{3}$ and $\boldsymbol{\mu} \in \mathscr{R}^{3}$, one obtains the following first-order nonlinear necessary conditions.

State Equations Are

$$
\dot{\boldsymbol{\sigma}}=\mathscr{H}_{\xi}=\mathbf{r},
$$

$$
\dot{\omega}=\mathscr{H}_{\mu}=\mathbf{f},
$$

where $(\cdot)_{\zeta} \triangleq \partial(\cdot) / \partial \zeta$ for a generic variable $\zeta$.

Costate Equations Are

$$
\begin{gathered}
\dot{\xi}=-\mathscr{H}_{\boldsymbol{\sigma}}=-\mathbf{r}_{\boldsymbol{\sigma}}^{T} \boldsymbol{\xi} \\
\dot{\boldsymbol{\mu}}=-\mathscr{H}_{\boldsymbol{\omega}}=-\mathbf{r}_{\boldsymbol{\omega}}^{T} \boldsymbol{\xi}-\mathbf{f}_{\boldsymbol{\omega}}^{T} \boldsymbol{\mu},
\end{gathered}
$$

where

$$
\begin{gathered}
\mathbf{f}_{\boldsymbol{\omega}}=\left[\begin{array}{ccc}
0 & \frac{J_{2}-J_{3}}{J_{1}} \omega_{3} & \frac{J_{2}-J_{3}}{J_{1}} \omega_{2} \\
\frac{J_{3}-J_{1}}{J_{2}} \omega_{3} & 0 & \frac{J_{3}-J_{1}}{J_{2}} \omega_{1} \\
\frac{J_{1}-J_{2}}{J_{3}} \omega_{2} & \frac{J_{1}-J_{2}}{J_{3}} \omega_{1} & 0
\end{array}\right], \quad \mathbf{r}_{\boldsymbol{\omega}}=\frac{1}{4}[B(\boldsymbol{\sigma})] \\
\mathbf{r}_{\boldsymbol{\sigma}}=\frac{1}{2}\left[\begin{array}{ccc}
\sigma_{1} \omega_{1}+\sigma_{2} \omega_{2}+\sigma_{3} \omega_{3} & \omega_{3}-\sigma_{2} \omega_{1}+\sigma_{1} \omega_{2} & \sigma_{1} \omega_{3}-\sigma_{3} \omega_{1}-\omega_{2} \\
\sigma_{2} \omega_{1}-\omega_{3}-\sigma_{1} \omega_{2} & \sigma_{1} \omega_{1}+\sigma_{2} \omega_{2}+\sigma_{3} \omega_{3} & \omega_{1}-\sigma_{3} \omega_{2}+\sigma_{2} \omega_{3} \\
-\sigma_{1} \omega_{3}+\sigma_{3} \omega_{1}+\omega_{2} & \sigma_{3} \omega_{2}-\omega_{1}-\sigma_{2} \omega_{3} & \sigma_{1} \omega_{1}+\sigma_{2} \omega_{2}+\sigma_{3} \omega_{3}
\end{array}\right]
\end{gathered}
$$

\section{Stationarity Condition Is}

$$
0=\mathscr{H}_{\mathbf{u}}=\mathscr{L}_{\mathbf{u}}+\mathbf{f}_{\mathbf{u}}^{T} \boldsymbol{\mu}=\mathbf{u}+P^{T} J^{-1} \boldsymbol{\mu} .
$$

Given the fixed initial time $t_{0}$ and final time $t_{f}$, the initial states $\boldsymbol{\sigma}\left(t_{0}\right)$ and $\boldsymbol{\omega}\left(t_{0}\right)$, and the final states $\boldsymbol{\sigma}\left(t_{f}\right)$ and $\boldsymbol{\omega}\left(t_{f}\right)$, there are no extra boundary conditions. These fixed terminal boundary conditions define a classical two-point boundaryvalue problem.

3.2. Optimal Control-Rate Formulation. We seek a solution of (3), (6), and (9) satisfying the terminal boundary conditions in (10) and

$$
\mathbf{u}\left(t_{0}\right)=\mathbf{u}_{0}, \quad \mathbf{u}\left(t_{f}\right)=\mathbf{u}_{f},
$$

where the 16 members of (10) and (16) are prescribed constants characterizing the attitude, angular velocity, and control torque at the initial and final times. Defining the Hamiltonian for the system

$$
\mathscr{H}=\mathscr{L}+\boldsymbol{\xi}^{T} \mathbf{r}+\boldsymbol{\mu}^{T} \mathbf{f}+\boldsymbol{\eta}^{T} \mathbf{g},
$$

where the loss function $\mathscr{L}=\dot{\mathbf{u}}^{T} \dot{\mathbf{u}} / 2$ and the Lagrange multiplier is $\boldsymbol{\eta} \in \mathscr{R}^{2}$, one obtains the following first-order nonlinear necessary conditions.

\section{State Equations Are}

$$
\begin{aligned}
& \dot{\boldsymbol{\sigma}}=\mathscr{H}_{\xi}=\mathbf{r}, \\
& \dot{\boldsymbol{\omega}}=\mathscr{H}_{\mu}=\mathbf{f}, \\
& \dot{\mathbf{u}}=\mathscr{H}_{\eta}=\mathbf{g} .
\end{aligned}
$$

Costate Equations Are

$$
\begin{gathered}
\dot{\boldsymbol{\xi}}=-\mathscr{H}_{\boldsymbol{\sigma}}=-\mathbf{r}_{\boldsymbol{\sigma}}^{T} \boldsymbol{\xi}, \\
\dot{\boldsymbol{\mu}}=-\mathscr{H}_{\boldsymbol{\omega}}=-\mathbf{r}_{\boldsymbol{\omega}}^{T} \boldsymbol{\xi}-\mathbf{f}_{\boldsymbol{\omega}}^{T} \boldsymbol{\mu}, \\
\dot{\boldsymbol{\eta}}=-\mathscr{H}_{\mathbf{u}}=-\mathbf{f}_{\mathbf{u}}^{T} \boldsymbol{\mu}=-P^{T} J^{-1} \boldsymbol{\mu} .
\end{gathered}
$$

Stationarity Condition Is

$$
0=\mathscr{H}_{\overline{\mathbf{u}}}=\mathscr{L}_{\overline{\mathbf{u}}}+\mathbf{g}_{\overline{\mathbf{u}}}^{T} \boldsymbol{\eta}=\overline{\mathbf{u}}+\boldsymbol{\eta} .
$$

As described in optimal control formulation, no additional boundary conditions exist.

\section{Problem Formulation for Failure Control}

For the underactuated system, a concept of sequential control is introduced to avoid a control input about the failed control axis. A sequential Euler angle transformation [23] is used for determining the MRPs at the switch time. Assuming that an Euler angle rotation sequence is selected that avoids the failed actuator axis, one seeks to design two successive submaneuvers where the maneuver times are unknown. At the interior switch time, the angular velocity is set to zero to avoid cross-coupling term's effect. These control design assumptions guarantee that the constraints for the states at the interior switch time are perfectly known.

After developing a strategy for carrying out two successive submaneuvers, the switch time must be specified. Failure to solve the optimal switch time leads to large penalties in performance indices, which indicates poor maneuver performance. 
4.1. Switch-Time Boundary Conditions. A free interior switch time $t_{s}$ is introduced for changing the control actuator being used; the following unknown boundary conditions are introduced:

$$
\mathscr{H}\left(t_{s}\right)=\mathscr{H}(t)=\text { constant }
$$

since the Hamiltonian is not an explicit function of time.

Because the unknown switch time is defined, a multiple shooting method [22] is applied to find a suboptimal solution, where the interior point condition [24] is given by

$$
N\left(\mathbf{z}\left(t_{s}\right)\right)=0,
$$

where $\mathbf{z}$ is the total states. The interior point condition yields the following two additional boundary conditions that define the suboptimal solution

$$
\begin{gathered}
\boldsymbol{\kappa}^{T}\left(t_{s}^{+}\right)=\boldsymbol{\kappa}^{T}\left(t_{s}^{-}\right)-\left.\boldsymbol{\alpha}^{T} N_{\mathbf{z}}\right|_{t_{s}}, \\
\mathscr{H}\left(t_{s}^{+}\right)=\mathscr{H}\left(t_{s}^{-}\right),
\end{gathered}
$$

where $\boldsymbol{\kappa}$ is the total Lagrange multipliers associated with the total states and $\boldsymbol{\alpha}$ is the another Lagrange multiplier describing the maneuver switch-time jump conditions.

\subsection{Optimal Control Formulation for Failure Control. An}

Euler angle transformation algorithm [23] is used to define the boundary conditions for the MRPs. Unlike the normal control, the MRPs at the switch time need to be determined. The boundary conditions for the MRPs are handled by using an Euler angle rotation sequence that avoids the failed control actuator axis.

To formulate the mathematical structure for the problem, one now collects all the problem unknowns and constraint conditions as follows.

19 Unknowns Are

$$
\begin{gathered}
@ t_{0}: \boldsymbol{\xi}\left(t_{0}\right) \in \mathscr{R}^{3}, \quad \boldsymbol{\mu}\left(t_{0}\right) \in \mathscr{R}^{3}, \\
@ t_{s}: \boldsymbol{\alpha}_{1} \in \mathscr{R}^{3}, \quad \boldsymbol{\alpha}_{2} \in \mathscr{R}^{3}, \quad t_{s} \in \mathscr{R}^{1}, \\
@ t_{f}: \boldsymbol{\xi}\left(t_{f}\right) \in \mathscr{R}^{3}, \quad \boldsymbol{\mu}\left(t_{f}\right) \in \mathscr{R}^{3} .
\end{gathered}
$$

19 Constraints Are

$$
\begin{gathered}
@ t_{s}: \boldsymbol{\sigma}\left(t_{s}^{-}\right)=\boldsymbol{\sigma}_{t_{s}}, \quad \boldsymbol{\omega}\left(t_{s}^{-}\right)=\boldsymbol{\omega}_{t_{s}}, \\
\boldsymbol{\sigma}\left(t_{s}^{+}\right)=\boldsymbol{\sigma}_{t_{s}}, \quad \boldsymbol{\omega}\left(t_{s}^{+}\right)=\boldsymbol{\omega}_{t_{s}}, \\
\boldsymbol{\xi}\left(t_{s}^{-}\right)=\boldsymbol{\xi}\left(t_{s}^{+}\right)+\boldsymbol{\alpha}_{3}, \\
\boldsymbol{\mu}\left(t_{s}^{-}\right)=\boldsymbol{\mu}\left(t_{s}^{+}\right)+\boldsymbol{\alpha}_{4}, \quad \mathscr{H}\left(t_{s}^{-}\right)=\mathscr{H}\left(t_{s}^{+}\right) .
\end{gathered}
$$

The constraints in (25) are particularly challenging because jump conditions govern the optimality of the resulting solutions. The problem is characterized by both high dimension and nonlinearity, which makes it critically important to develop useful approximate starting solutions. The unknowns in (24a), (24b), and (24c) and the constraints in (25) are enforced by iteratively solving (12a), (12b), (13a), (13b), and (15).
TABLE 1: Numerical simulation parameters.

\begin{tabular}{lcc}
\hline Parameter & Value & Unit \\
\hline Inertia of the spacecraft & $\operatorname{diag}[14.2,17.3,20.3]$ & $\mathrm{kg} \cdot \mathrm{m}^{2}$ \\
\hline Initial angular velocity & {$[0,0,0]^{T}$} & $\mathrm{deg} / \mathrm{s}$ \\
Initial control torque & {$[0,0]^{T}$} & $\mathrm{Nm}$ \\
Initial Euler angles (1-2-1 set) & {$[15,30,45]^{T}$} & $\mathrm{deg}$ \\
Initial MRPs & {$[0.263,0.1361,-0.037]^{T}$} & - \\
\hline Interior angular velocity & {$[0,0,0]^{T}$} & $\mathrm{deg} / \mathrm{s}$ \\
Interior control torque & {$[0,0]^{T}$} & $\mathrm{Nm}$ \\
Interior Euler angles (1-2-1 set) & {$[15,30,0]^{T}$} & $\mathrm{deg}$ \\
Interior MRPs & {$[0.064,0.131,0.017]^{T}$} & - \\
\hline Final angular velocity & {$[0,0,0]^{T}$} & $\mathrm{deg} / \mathrm{s}$ \\
Final control torque & {$[0,0]^{T}$} & $\mathrm{Nm}$ \\
Final Euler angles (1-2-1 set) & {$[0,0,0]^{T}$} & $\mathrm{deg}$ \\
Final MRPs & {$[0,0,0]^{T}$} & - \\
\hline
\end{tabular}

4.3. Optimal Control-Rate Formulation for Failure Control. To formulate the mathematical structure for the problem, one now collects all the problem unknowns and constraint conditions as follows.

25 Unknowns Are

$$
\begin{gathered}
@ t_{0}: \xi\left(t_{0}\right) \in \mathscr{R}^{3}, \quad \boldsymbol{\mu}\left(t_{0}\right) \in \mathscr{R}^{3}, \\
\boldsymbol{\eta}\left(t_{0}\right) \in \mathscr{R}^{2}, \\
@ t_{s}: \boldsymbol{\alpha}_{1} \in \mathscr{R}^{3}, \quad \boldsymbol{\alpha}_{2} \in \mathscr{R}^{3}, \\
\boldsymbol{\alpha}_{3} \in \mathscr{R}^{2}, \quad t_{s} \in \mathscr{R}^{1}, \\
@ t_{f}: \boldsymbol{\xi}\left(t_{f}\right) \in \mathscr{R}^{3}, \quad \boldsymbol{\mu}\left(t_{f}\right) \in \mathscr{R}^{3}, \\
\boldsymbol{\eta}\left(t_{f}\right) \in \mathscr{R}^{2} .
\end{gathered}
$$

25 Constraints Are

$$
\begin{gathered}
@ t_{s}: \boldsymbol{\sigma}\left(t_{s}^{-}\right)=\boldsymbol{\sigma}_{t_{s}}, \quad \boldsymbol{\omega}\left(t_{s}^{-}\right)=\boldsymbol{\omega}_{t_{s}}, \\
\mathbf{u}\left(t_{s}^{-}\right)=\mathbf{u}_{t_{s}}, \\
\boldsymbol{\sigma}\left(t_{s}^{+}\right)=\boldsymbol{\sigma}_{t_{s}}, \quad \boldsymbol{\omega}\left(t_{s}^{+}\right)=\boldsymbol{\omega}_{t_{s}}, \\
\mathbf{u}\left(t_{s}^{+}\right)=\mathbf{u}_{t_{s}}, \\
\boldsymbol{\xi}\left(t_{s}^{-}\right)=\boldsymbol{\xi}\left(t_{s}^{+}\right)+\boldsymbol{\alpha}_{4}, \quad \boldsymbol{\mu}\left(t_{s}^{-}\right)=\boldsymbol{\mu}\left(t_{s}^{+}\right)+\boldsymbol{\alpha}_{5}, \\
\boldsymbol{\eta}\left(t_{s}^{-}\right)=\boldsymbol{\eta}\left(t_{s}^{+}\right)+\boldsymbol{\alpha}_{6}, \quad \mathscr{H}\left(t_{s}^{-}\right)=\mathscr{H}\left(t_{s}^{+}\right) .
\end{gathered}
$$

The unknowns of (26a), (26b), and (26c) and the constraints of (27) are enforced by iteratively solving (18a), (18b), (18c), (19a), (19b), (19c), and (20).

\section{Numerical Results}

The numerical simulation parameters are listed in Table 1. For asymmetric rigid spacecraft, a diagonal moment of inertia 

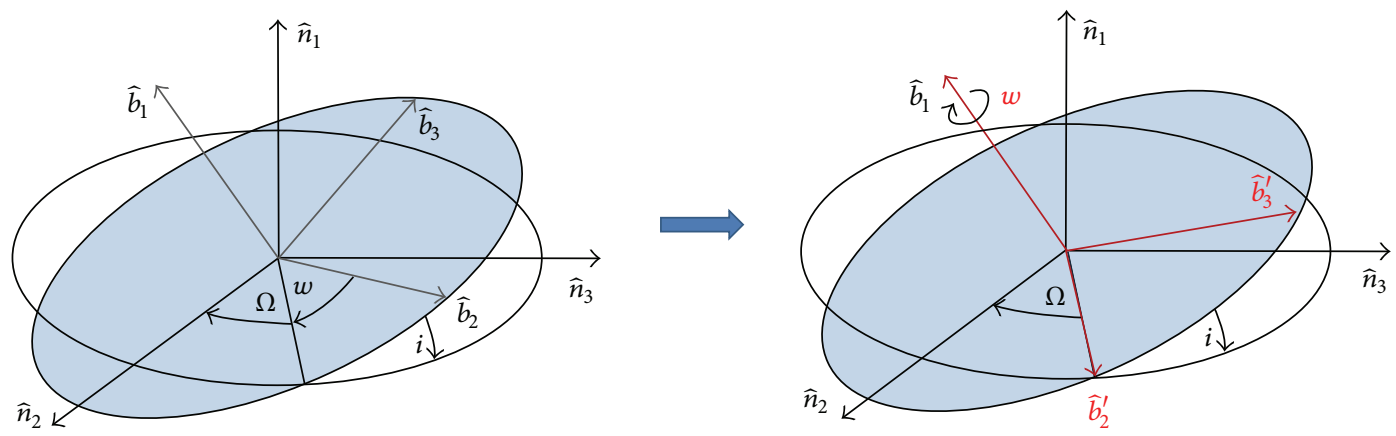

FIGURE 1: Initial attitude movement to torque available plane using torque available axis rotation.

TABle 2: Comparison between guesses and found values (torquerate minimization).

\begin{tabular}{lcc}
\hline Parameter & Guess & Found value \\
\hline $\boldsymbol{\mu}\left(t_{0}\right)$ & {$[0,0,0]^{T}$} & {$[2.040,0,0]^{T}$} \\
$\boldsymbol{\xi}\left(t_{0}\right)$ & {$[0,0,0]^{T}$} & {$[1.117,-0.001,-0.310]^{T}$} \\
$\boldsymbol{\eta}\left(t_{0}\right)$ & {$[0,0]^{T}$} & {$[0.310,0]^{T}$} \\
\hline $\boldsymbol{\mu}\left(t_{f}\right)$ & {$[0,0,0]^{T}$} & {$[-2.898,-0.710,-115.523]^{T}$} \\
$\boldsymbol{\xi}\left(t_{f}\right)$ & {$[0,0,0]^{T}$} & {$[2.528,3.429,-7.982]^{T}$} \\
$\boldsymbol{\eta}\left(t_{f}\right)$ & {$[0,0]^{T}$} & {$[0.310,-0.003]^{T}$} \\
\hline $\boldsymbol{\alpha}_{1}$ & {$[0,0,0]^{T}$} & {$[-5.286,-1.983,148.211]^{T}$} \\
$\boldsymbol{\alpha}_{2}$ & {$[0,0,0]^{T}$} & {$[-3.354,-2.203,6.941]^{T}$} \\
$\boldsymbol{\alpha}_{3}$ & {$[0,0]^{T}$} & {$[0.146,-0.263]^{T}$} \\
\hline$t_{s}$ & 15 & 12.930 \\
\hline
\end{tabular}

tensor is assumed to model the spacecraft, where all values of the inertia are different.

Numerical simulations are performed for both nominal (3-axis controls) and failure control cases (2-axis controls). For the failure control simulation case, $u_{1}$ and $u_{2}$ are only available. Using the Euler transformation, the given initial attitude can be transformed into two possible sets of expression, such as (1-2-1) and (2-1-2). In this paper, the (1-2-1) set is studied, and a trend of first single-axis maneuver is shown in Figure 1. Future work will develop algorithms for optimally selecting the Euler angle rotation sequence.

The unknown Lagrange multipliers are selected arbitrarily, and the unknown switch times are initialized by assuming half of the total simulation time. With this initial guesses, the Lagrange multipliers and switch time are found and listed in Tables 3 and 2 .

The simulation results for minimizing control torques are shown in Figures 2-5. Figure 2 shows that all the states meet the terminal and interior conditions using only two control torques. Since a single-axis maneuver is conducted until the switch time, we observe linear control time histories. Then, two control torques are utilized for reorientation. Note that discontinuous control time histories are obtained for torque minimization case. Figure 3 presents time histories of Lagrange multipliers associated with the states.

The results among the normal control, failure control using two successive maneuvers, and failure control using
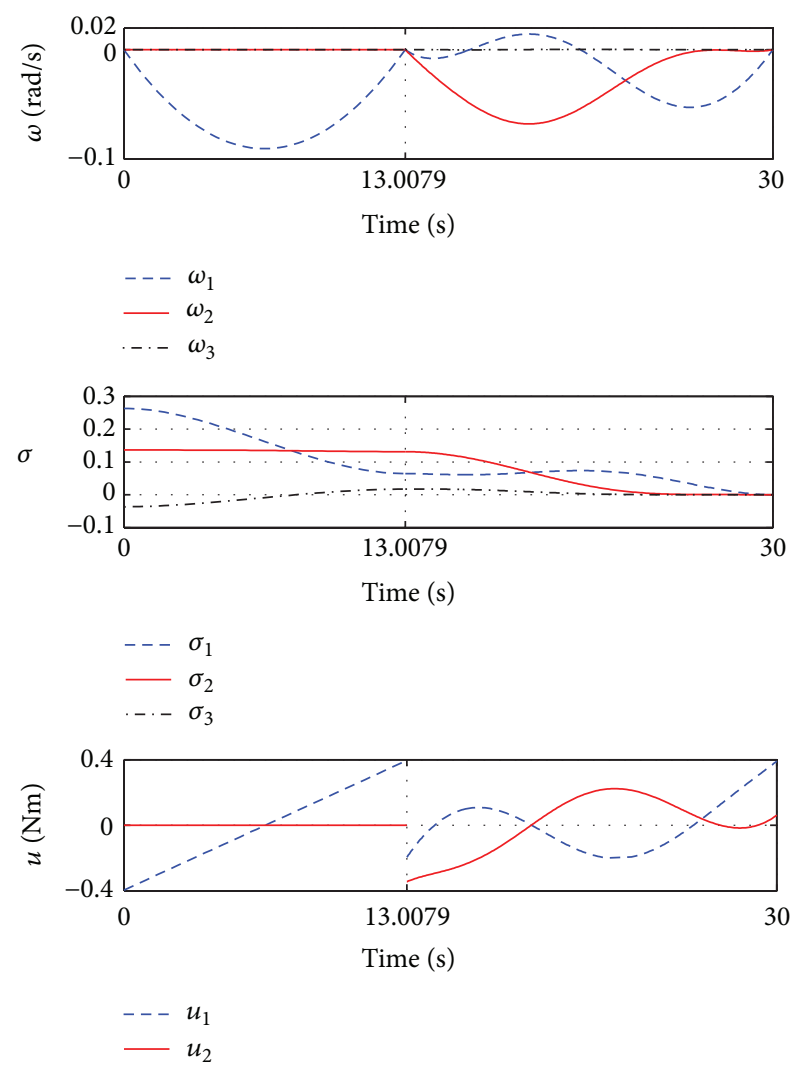

FIgURE 2: Time trajectory of states (torque minimization).

three successive maneuvers are compared. Figure 4 presents the Hamiltonian and performance index time histories. As defined in (21), it shows that Hamiltonian is constant over time regardless of normal and failure controls. For the normal control, the performance index value at final time indicates the torque consumption. For the failure control, the sum of the performance index value at switch times and final time indicates the torque consumption. Figure 5 presents the principal angle time histories. We observe that the principal angle path using three successive submaneuvers is the longest. Thus we can expect that the two-successive-submaneuver strategy has better performance than the three-successivesubmaneuver strategy. 

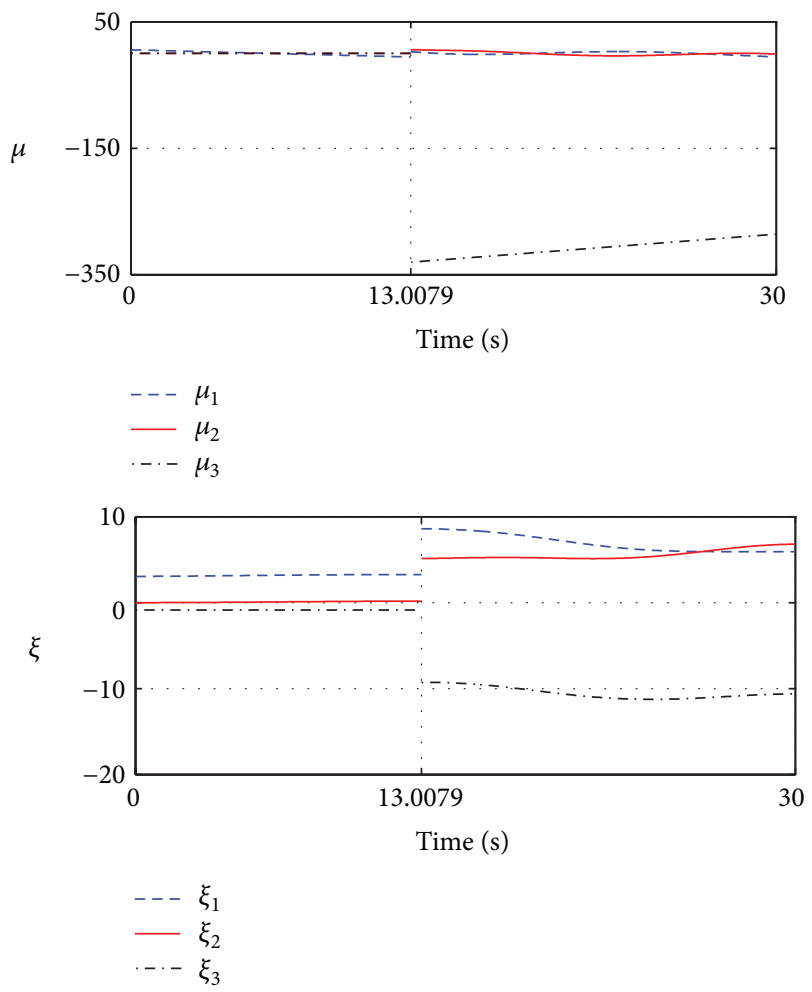

FIGURE 3: Time trajectory of costates (torque minimization).

TABLE 3: Comparison between guesses and found values (torque minimization).

\begin{tabular}{lcc}
\hline Parameter & Guess & Found value \\
\hline $\boldsymbol{\mu}\left(t_{0}\right)$ & {$[0,0,0]^{T}$} & {$[5.616,0,0]^{T}$} \\
$\boldsymbol{\xi}\left(t_{0}\right)$ & {$[0,0,0]^{T}$} & {$[3.056,-0.004,-0.849]^{T}$} \\
\hline $\boldsymbol{\mu}\left(t_{f}\right)$ & {$[0,0,0]^{T}$} & {$[-5.549,-1.053,-285.835]^{T}$} \\
$\boldsymbol{\xi}\left(t_{f}\right)$ & {$[0,0,0]^{T}$} & {$[5.929,6.798,-10.628]^{T}$} \\
\hline $\boldsymbol{\alpha}_{1}$ & {$[0,0,0]^{T}$} & {$[-8.389,-5.949,329.183]^{T}$} \\
$\boldsymbol{\alpha}_{2}$ & {$[0,0,0]^{T}$} & {$[-5.322,-4.968,8.416]^{T}$} \\
\hline$t_{s}$ & 15 & 13.008 \\
\hline
\end{tabular}

The simulation results for minimizing control torque rates are shown in Figures 6-9. Figure 6 shows that all the states meet the terminal and interior conditions using only two control torques. Even though a single-axis maneuver is conducted until the switch time, different control time histories are obtained. This is because control rates are minimized instead of control torques. Also, it leads to continuous control time histories. Figure 7 presents time histories of Lagrange multipliers associated with the state.

Similarly, constant Hamiltonian time histories are obtained in Figure 8. Like the control torque minimization results of the principal angle path, the three-successivesubmaneuver result has the longest path in Figure 9. For comparing torque consumptions according to the usage of control rate as a performance index, $\mathscr{G}^{\overline{\mathbf{u}}} \triangleq(1 / 2) \int_{t_{0}}^{t_{f}} \mathbf{u}^{T} \mathbf{u} \mathrm{d} t$ is calculated. For both torque and torque-rate minimization cases, torque consumptions are compared and listed in Table 4.
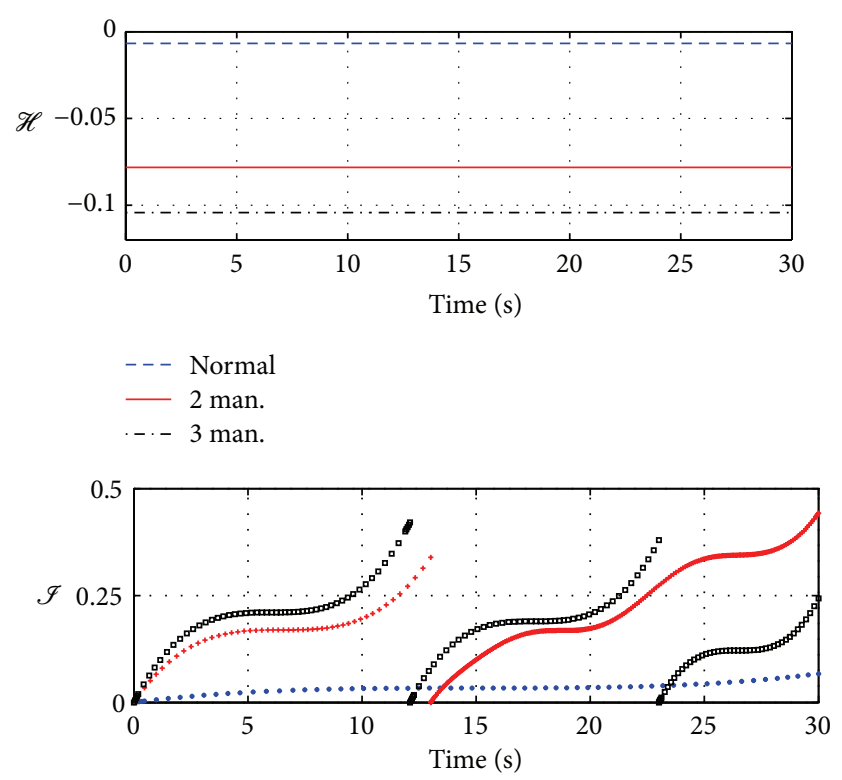

- Normal

+2 man.

- 3 man.

FIGURE 4: Time trajectory of Hamiltonian and performance index (torque minimization).

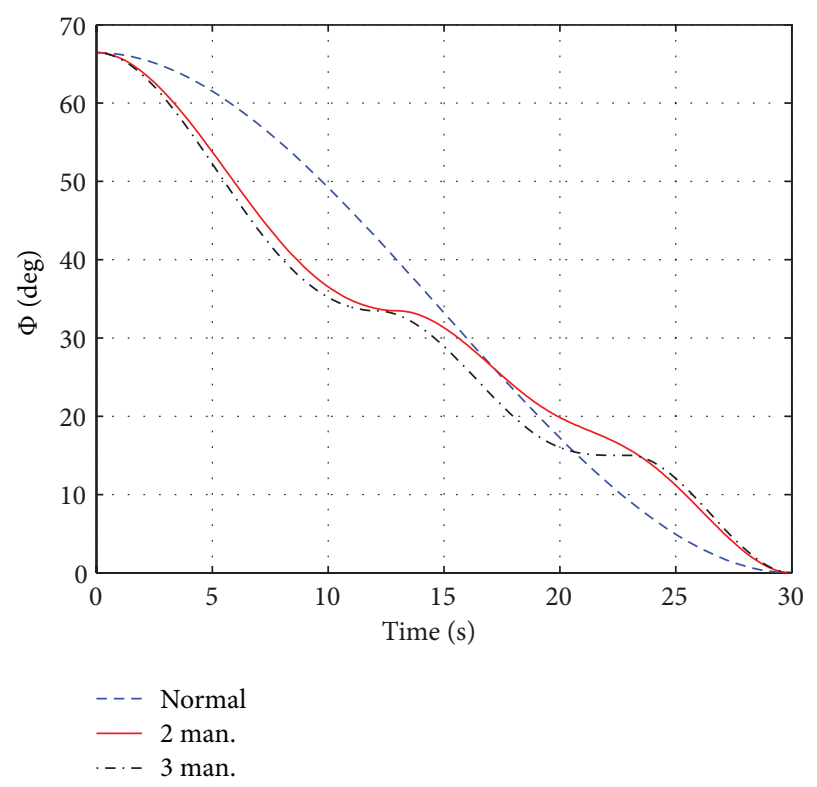

FIGURE 5: Time trajectory of principal angle (torque minimization).

As shown in Table 4, the two-successive-submaneuver strategy requires less torque than the three-successivesubmaneuver one.

\section{Conclusion}

The classic spacecraft maneuver problem is generalized to handle the special case that an actuator failure alters the 


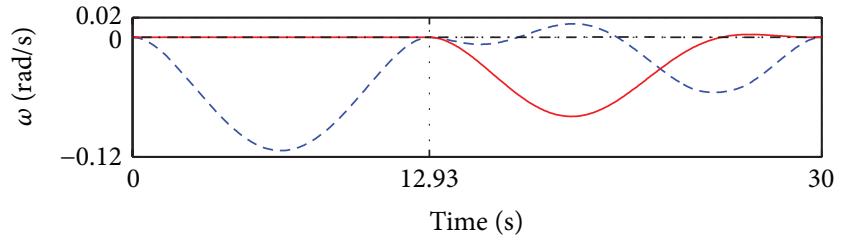

$---\omega_{1}$

$-\omega_{2}$

$\cdot-\cdot \omega_{3}$

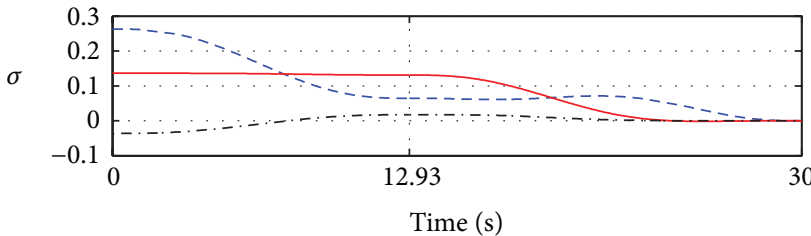

$$
\begin{array}{ll}
--- & \sigma_{1} \\
- & \sigma_{2} \\
-\cdot- & \sigma_{3}
\end{array}
$$

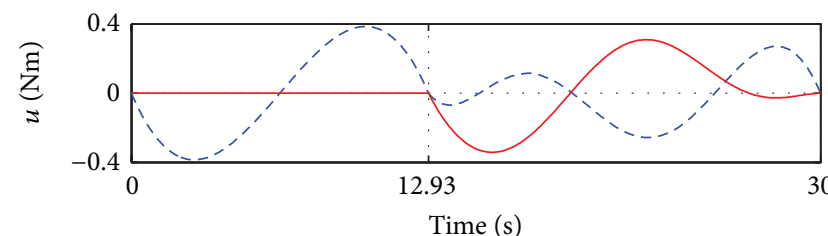

$$
---u_{1}
$$

FIGURE 6: Time trajectory of states (torque-rate minimization).

$\mu$

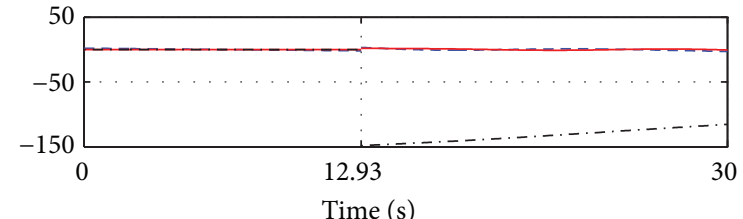

$---\mu_{1}$

$-\mu_{2}$

$\cdot-\cdot-\mu_{3}$

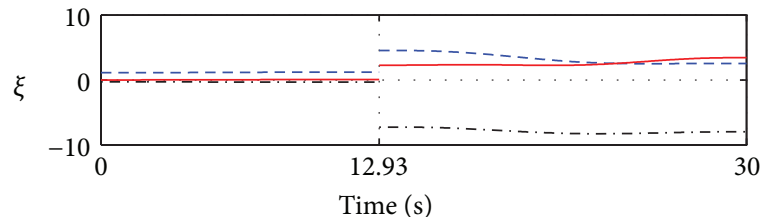

$--\xi_{1}$

$-\xi_{2}$

$--\xi_{3}$

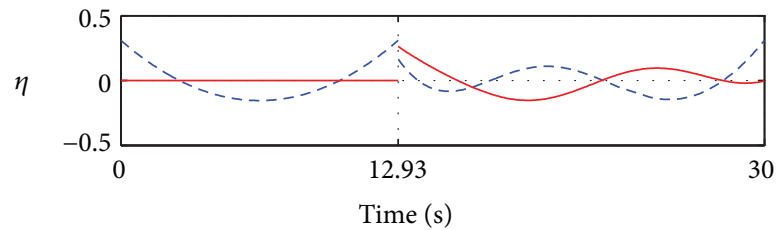

$---\eta_{1}$

$-\eta_{2}$

FIgURE 7: Time trajectory of costates (torque-rate minimization).

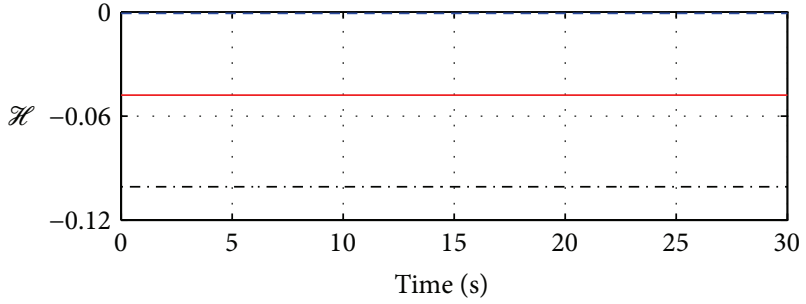

$$
\begin{aligned}
& -- \text { Normal } \\
& -2 \text { man. } \\
& -.-3 \text { man. }
\end{aligned}
$$

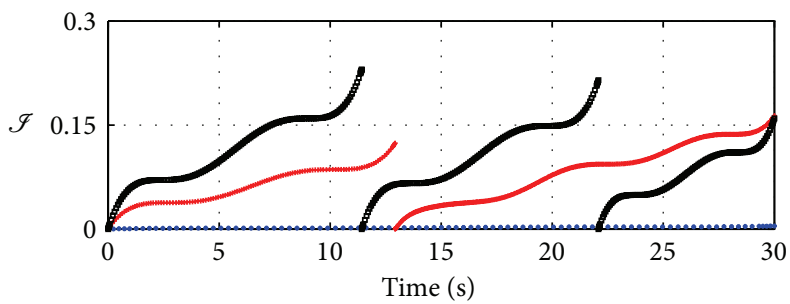

- Normal

+ 2 man.

- 3 man.

FIgURE 8: Time trajectory of Hamiltonian and performance index (torque-rate minimization).

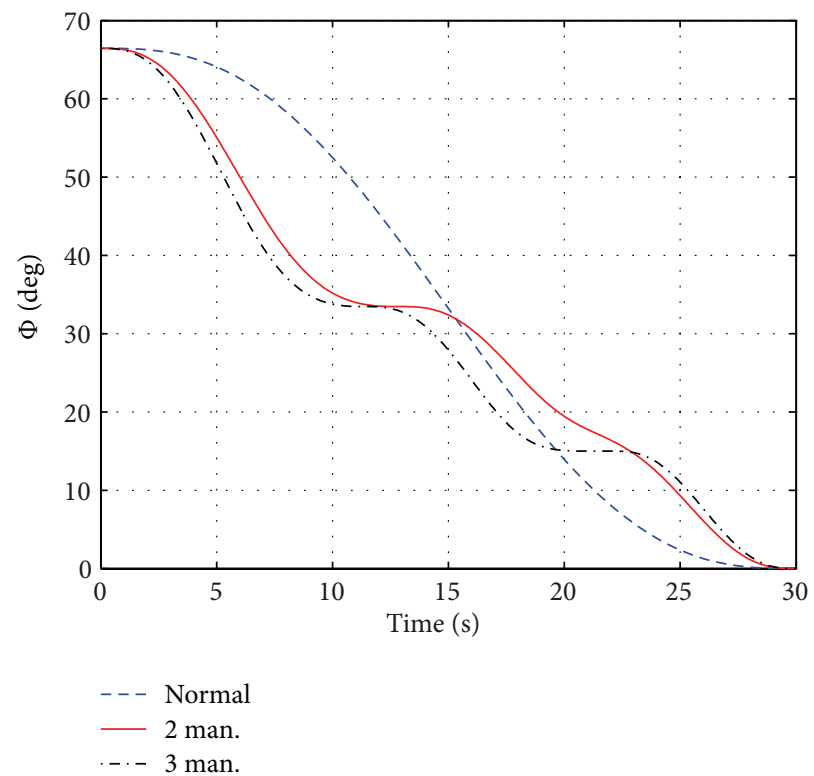

FIGURE 9: Time trajectory of principal angle (torque-rate minimization).

TABLE 4: Torque consumption comparison.

\begin{tabular}{lccc}
\hline Observation & Normal & 2 man. (ratio) & 3 man. (ratio) \\
\hline $\mathcal{J}^{\mathrm{u}}$ & 0.0671 & $0.7820(11.6502)$ & $1.0432(15.5414)$ \\
$\mathscr{G}^{\mathrm{u}}$ & 0.0959 & $1.0525(10.9700)$ & $1.5348(15.9965)$ \\
\hline
\end{tabular}

hardware capabilities available for repointing the vehicle. A key objective is to maintain a suboptimal solution strategy 
even during the degraded hardware environment. A solution strategy is presented that enables two control inputs to complete the originally defined three-dimensional rigid body maneuver. Nonlinear necessary conditions are defined for carrying out a sequence of maneuvers. For a single-axis and simultaneous submaneuver, an unknown switch time must be recovered switching between the remaining control actuators. Two control formulations are presented. The first approach penalizes the control torques but is shown to lead to discontinuous control time histories. The second approach penalizes the control torque rates but is shown to eliminate the discontinuous control time histories, which is important for flexible body applications. The problem is defined by a nonlinear high-dimensional set of necessary conditions, which are iteratively solved by introducing a multiple shooting method. On comparing the performance indices for the nominal and failed actuator cases, it is demonstrated that the failed actuator case requires $\sim 20$-fold increase in computational cost. The proposed method is expected to be broadly useful for spacecraft applications that must deal with actuator failures on orbit, where optimized approaches are required for maintaining vehicle pointing goals. Future research will investigate developing more accurate starting guess solutions for reducing the number of nonlinear optimization iterations required.

\section{References}

[1] J. L. Junkins and J. D. Turner, "Optimal continuous torque attitude maneuvers," Journal of Guidance, Control, and Dynamics, vol. 3, no. 3, pp. 210-217, 1980.

[2] J. D. Turner and J. L. Junkins, "Optimal large-angle single axis rotational maneuvers of flexible spacecraft," Journal of Guidance, Control, and Dynamics, vol. 3, no. 6, pp. 578-585, 1980.

[3] J. L. Junkins and J. D. Turner, Optimal Spacecraft Rotational Maneuvers, vol. 3 of Studies in Astronautics, Elsevier, Amsterdam, The Netherlands, 1986.

[4] P. Tsiotras and J. M. Longuski, "A new parameterization of the attitude kinematics," Journal of the Astronautical Sciences, vol. 43, no. 3, pp. 243-262, 1995.

[5] E.-Y. Keraï, "Analyis of small time local controllability of the rigid body model," in Proceedings of the 3rd IFAC Symposium on System Structure and Control, pp. 597-602, Oxford, UK, 1995.

[6] R. W. Brockett, "Asymptotic stability and feedback stabilization," in Differential Geometric Control Theory, pp. 181-191, Birkhäuser, Cambridge, Mass, USA, 1983.

[7] P. Tsiotras, M. Corless, and J. M. Longuski, "A novel approach to the attitude control of axisymmetric spacecraft," Automatica, vol. 31, no. 8, pp. 1099-1112, 1995.

[8] P. Tsiotras, "Optimal regulation and passivity results for axisymmetric rigid bodies using two controls," Journal of Guidance, Control, and Dynamics, vol. 20, no. 3, pp. 457-463, 1997.

[9] P. Tsiotras and J. Luo, "Reduced effort control laws for underactuated rigid spacecraft," Journal of Guidance, Control, and Dynamics, vol. 20, no. 6, pp. 1089-1095, 1997.

[10] P. Tsiotras and J. Luo, "Stabilization and tracking of underactuated axisymmetric spacecraft with bounded control," in Proceedings of the 4th IFAC Symposium on Nonlinear Control
Systems Design, pp. 800-806, Pergamon Press, Enschede, The Netherlands, 1998.

[11] H. Shen and P. Tsiotras, "Time-optimal control of axisymmetric rigid spacecraft using two controls," Journal of Guidance, Control, and Dynamics, vol. 22, no. 5, pp. 682-694, 1999.

[12] P. Tsiotras and J. Luo, "Control of underactuated spacecraft with bounded inputs," Automatica, vol. 36, no. 8, pp. 1153-1169, 2000.

[13] P. Tsiotras and V. Doumtchenko, "Control of spacecraft subject to actuator failures: stateof-the-art and open problems," in The Richard H. Battin Astrodynamics Conference, pp. 1-21, American Astronautical Society, San Diego, Calif, USA, No. 00-264.

[14] P. Tsiotras and A. Schleicher, "Detumbling and partial attitude stabilization of a rigid spacecraft under actuator failure," in Proceedings of the AIAA Guidance, Navigation, and Control Conference and Exhibit, AIAA, Denver, Colo, USA, August 2000, No. 2000-4044.

[15] P. Morin, C. Samson, J.-B. Pomet, and Z.-P. Jiang, “Timevarying feedback stabilization of the attitude of a rigid spacecraft with two controls," Systems \& Control Letters, vol. 25, no. 5, pp. 375-385, 1995.

[16] J.-M. Coron and E.-Y. Keraï, "Explicit feedbacks stabilizing the attitude of a rigid spacecraft with two control torques," Automatica, vol. 32, no. 5, pp. 669-677, 1996.

[17] D. Kim and J. D. Turner, "3D spacecraft attitude control using two control inputs," in Proceedings of the Annual Technical Symposium, p. 26, AIAA, May 2012, No. A-3.3.

[18] D. Kim and J. D. Turner, "Spacecraft attitude control using two control torques," in Proceedings of the Jer-Nan Juang Astrodynamics Symposium, American Astronautical Society, College Station, Tex, USA, June 2012, No.AAS 12-640.

[19] D. Kim and J. D. Turner, "Suboptimal spacecraft maneuvers using two controls," Journal of Guidance, Control, and Dynamics.

[20] J. L. Junkins, Z. H. Rahman, and H. Bang, "Near-minimumtime control of distributed parameter systems. Analytical and experimental results," Journal of Guidance, Control, and Dynamics, vol. 14, no. 2, pp. 406-415, 1991.

[21] D. Kim, 3D maneuvers for asymmetric under-actuated rigid body [Ph.D. thesis], Texas A\&M University, 2013.

[22] H. G. Bock and K. J. Plitt, "A multiple shooting algorithm for direct solution of optimal problems," in Proceeedings of the 9th IFAC World Congress, pp. 242-247, IEEE Publications, Budapest, Hungary, 1984.

[23] H. Schaub and J. L. Junkins, Analytical Mechanics of Space Systems, AIAA, Reston, Va, USA, 2nd edition, 2009.

[24] H. J. Pesch, "A practical guide to the solution of real-life optimal control problems," Control and Cybernetics, vol. 23, no. 1-2, pp. 7-60, 1994. 


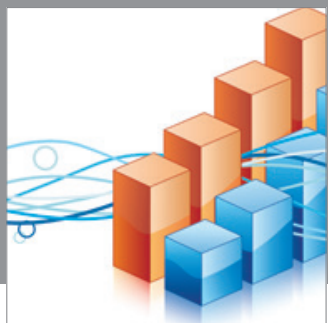

Advances in

Operations Research

mansans

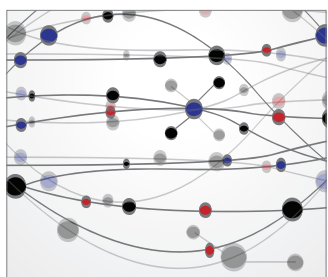

The Scientific World Journal
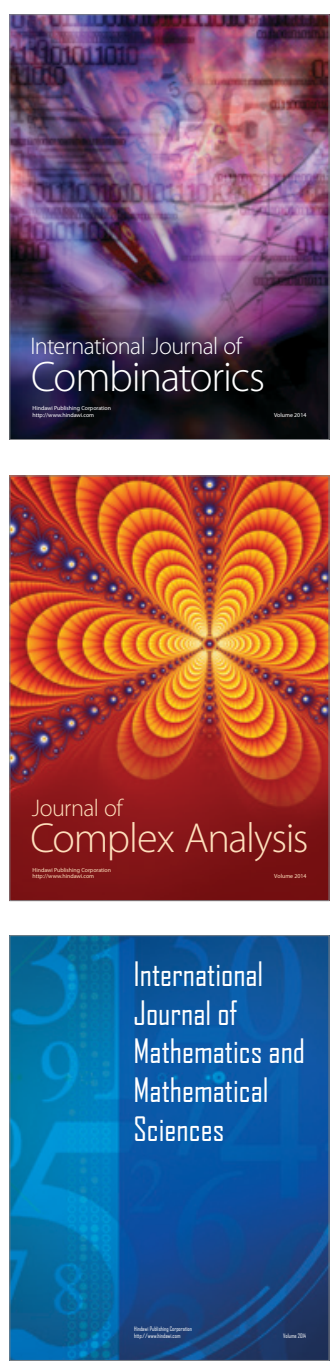
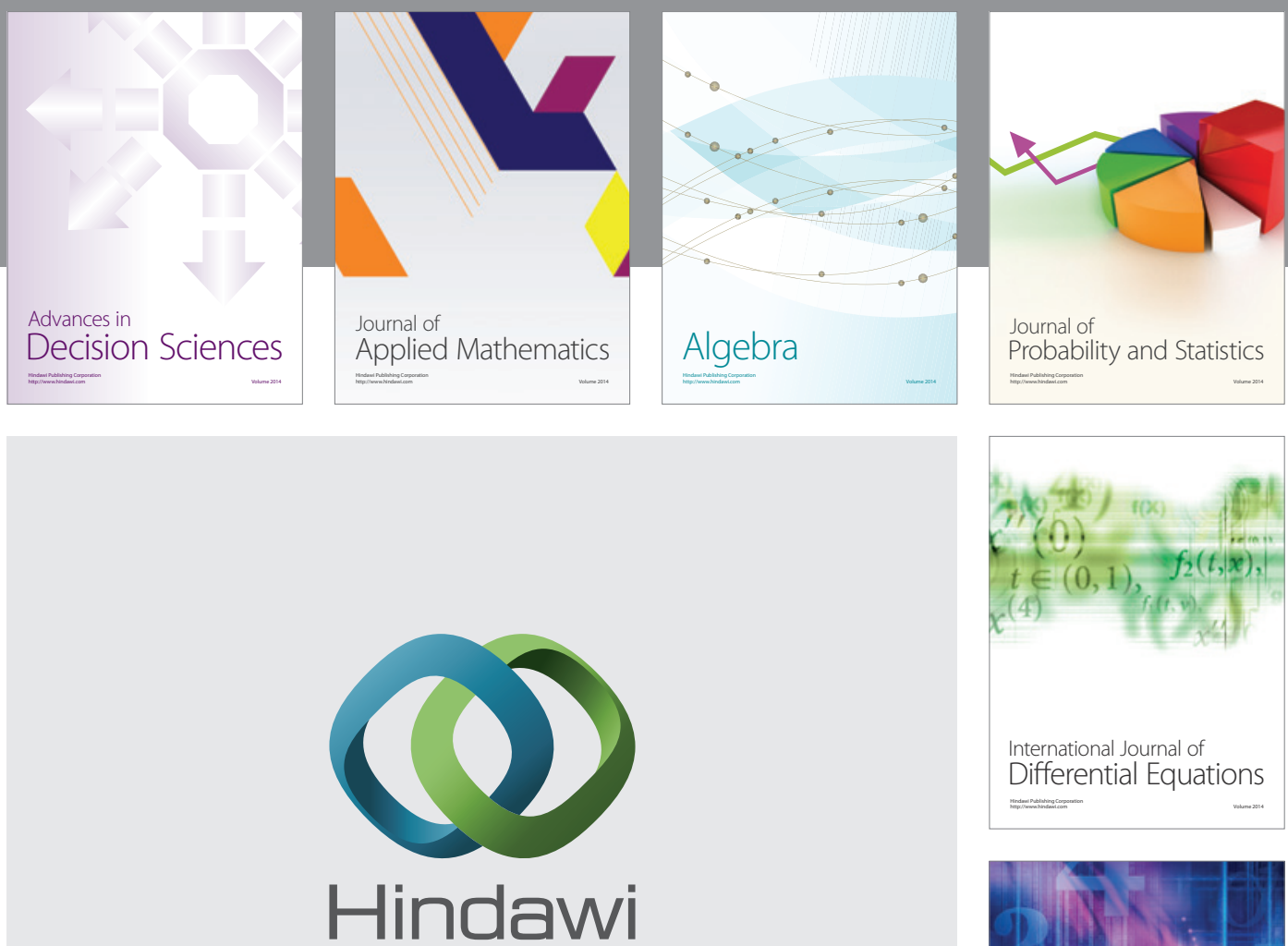

Submit your manuscripts at http://www.hindawi.com
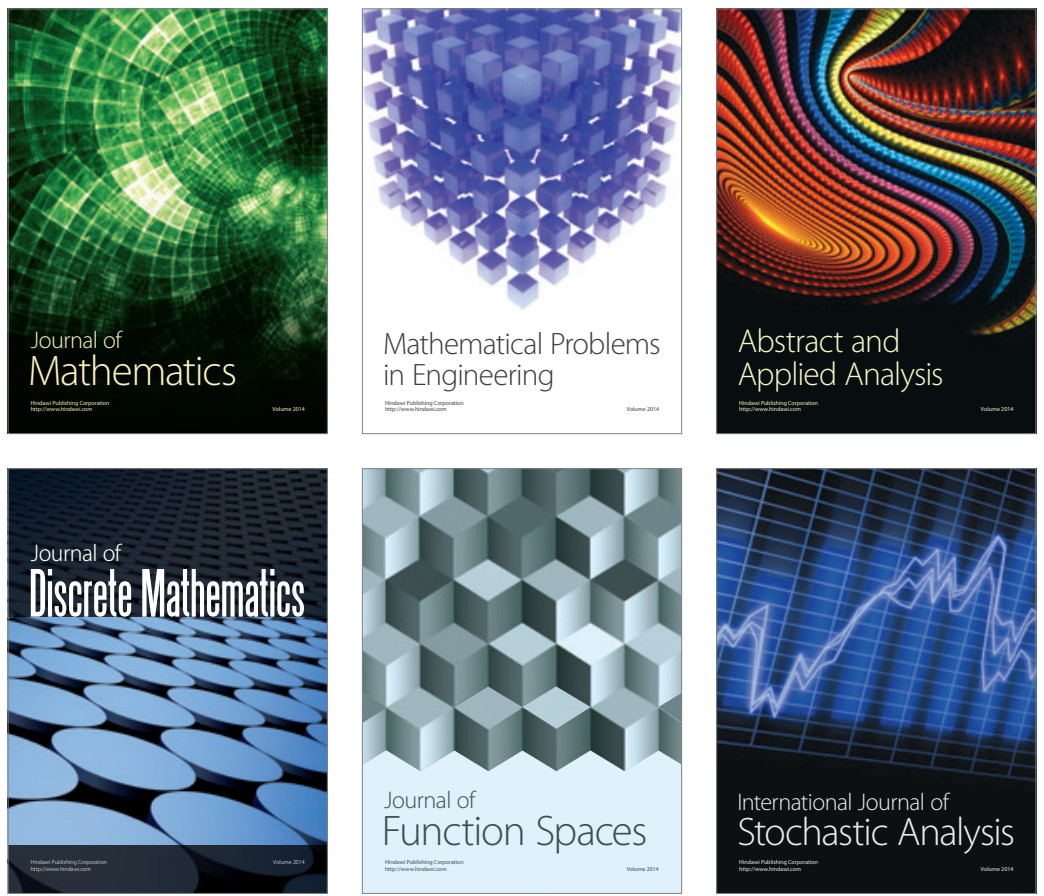

Journal of

Function Spaces

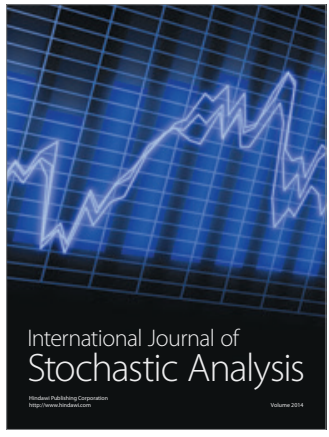

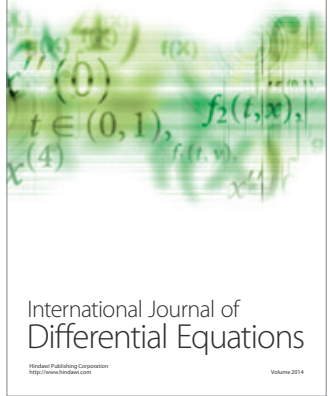
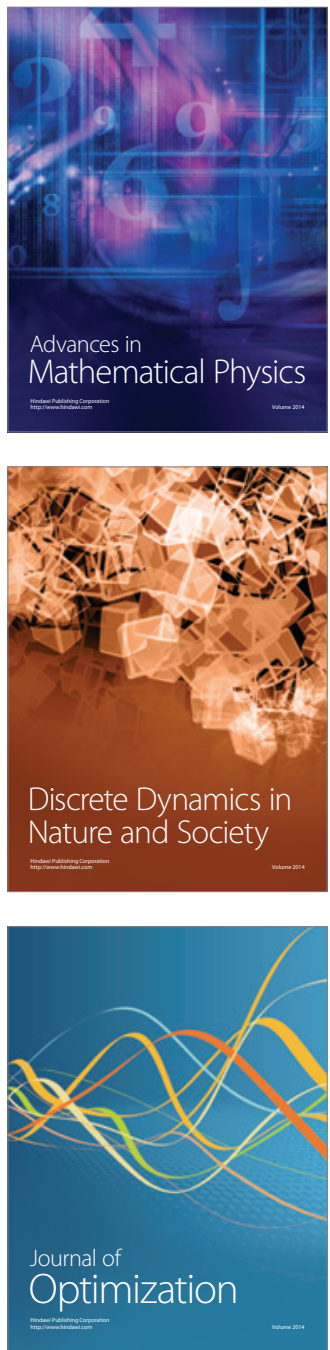